\title{
Fisher information and thermodynamic cost of near-equilibrium computation
}

(collaborators: Emanuele Crosato, Joseph Lizier Ramil Nigmatullin, Richard Spinney)

Prof. Mikhail Prokopenko Centre for Complex Systems Faculty of Engineering \& IT 


\section{Motivation...}

Chris Langton, "Computation at the edge of chaos: Phase transitions and emergent computation" (1991):

- how can emergence of computation be explained in a dynamic setting?

- how is it related to complexity of the system in point?

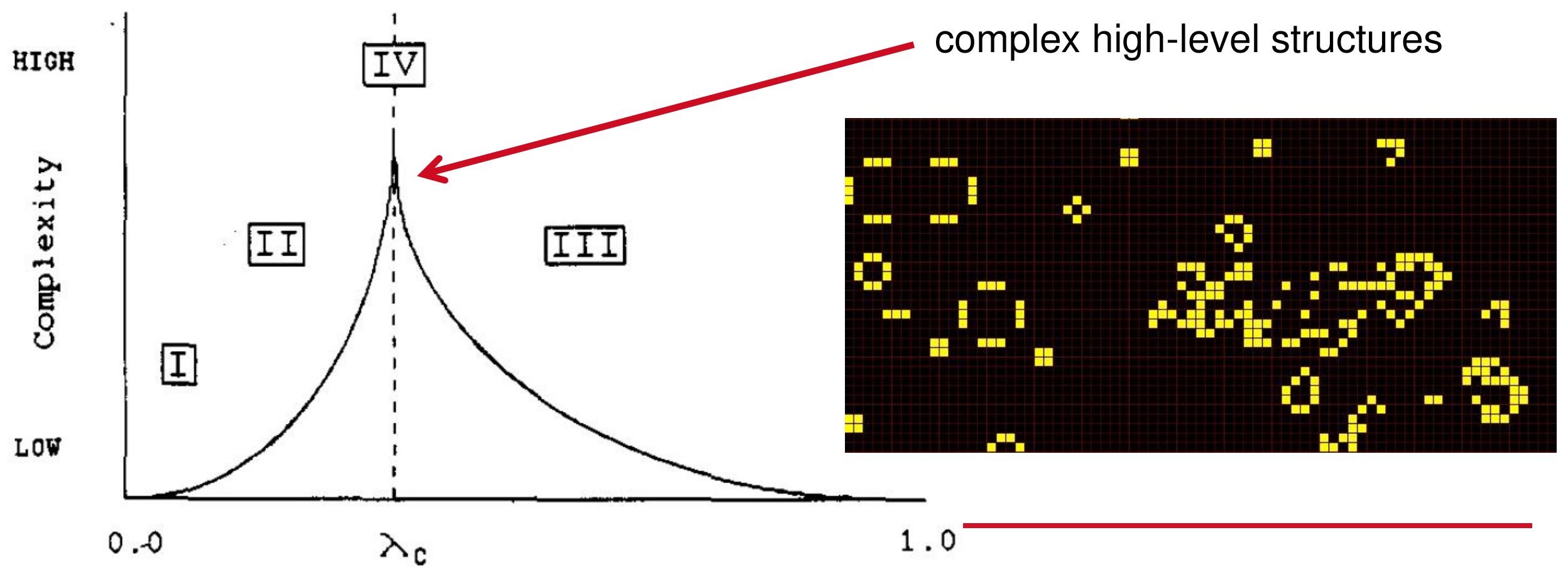




\section{Outline (...back to thermodynamics)}

$>$ Edge of chaos, criticality and phase transitions

$>$ Sensitivity of computation (Fisher information)

$>$ Uncertainty of computation (entropy curvature)

$>$ Example: collective motion 


\section{Phase transitions and order parameters}

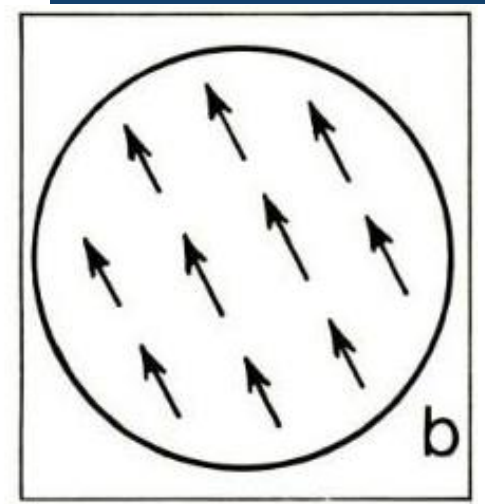

Magnetization from mean field approximation
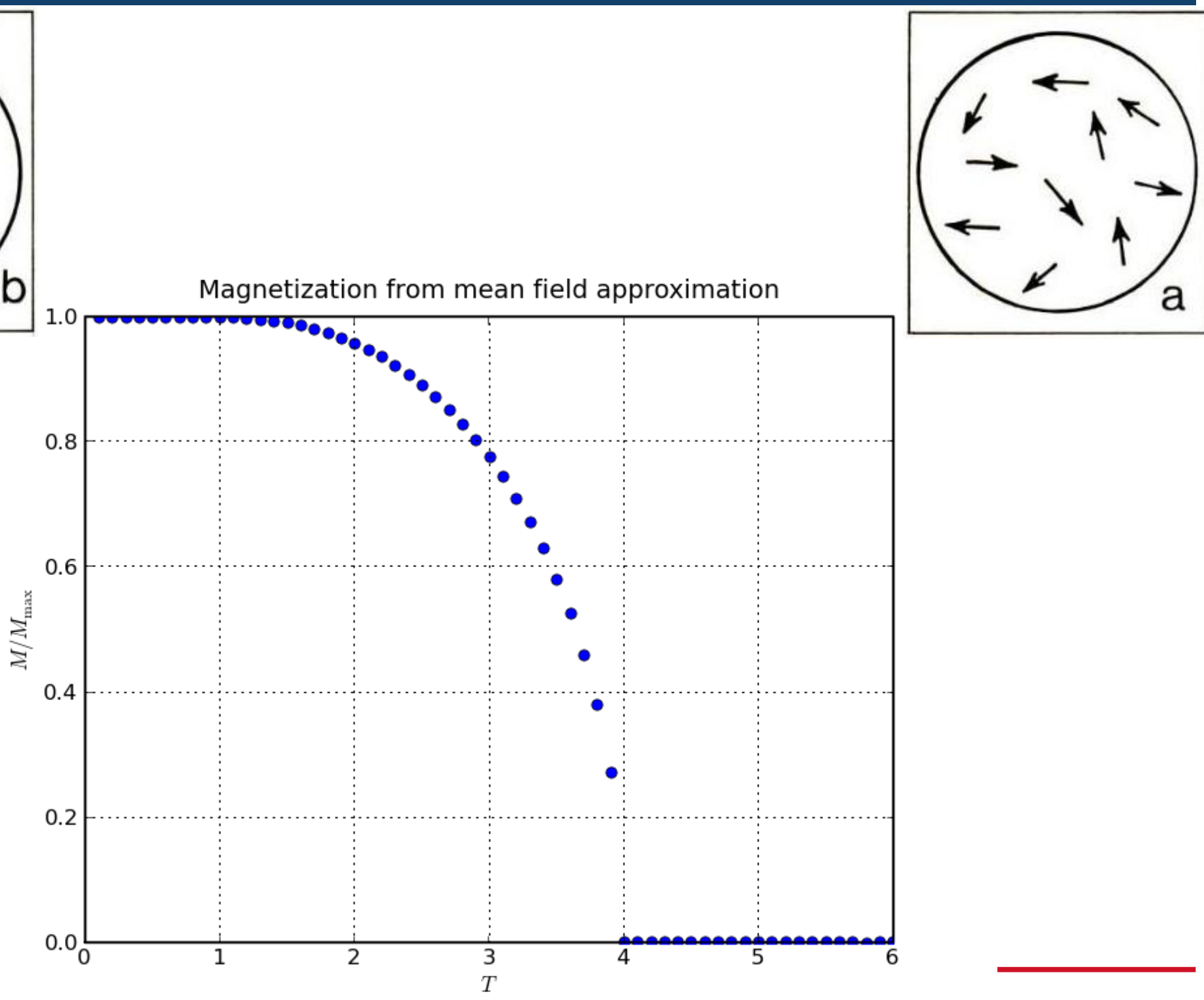


\section{Derivative of order parameter (divergence)}

$K$ Binder (1987)

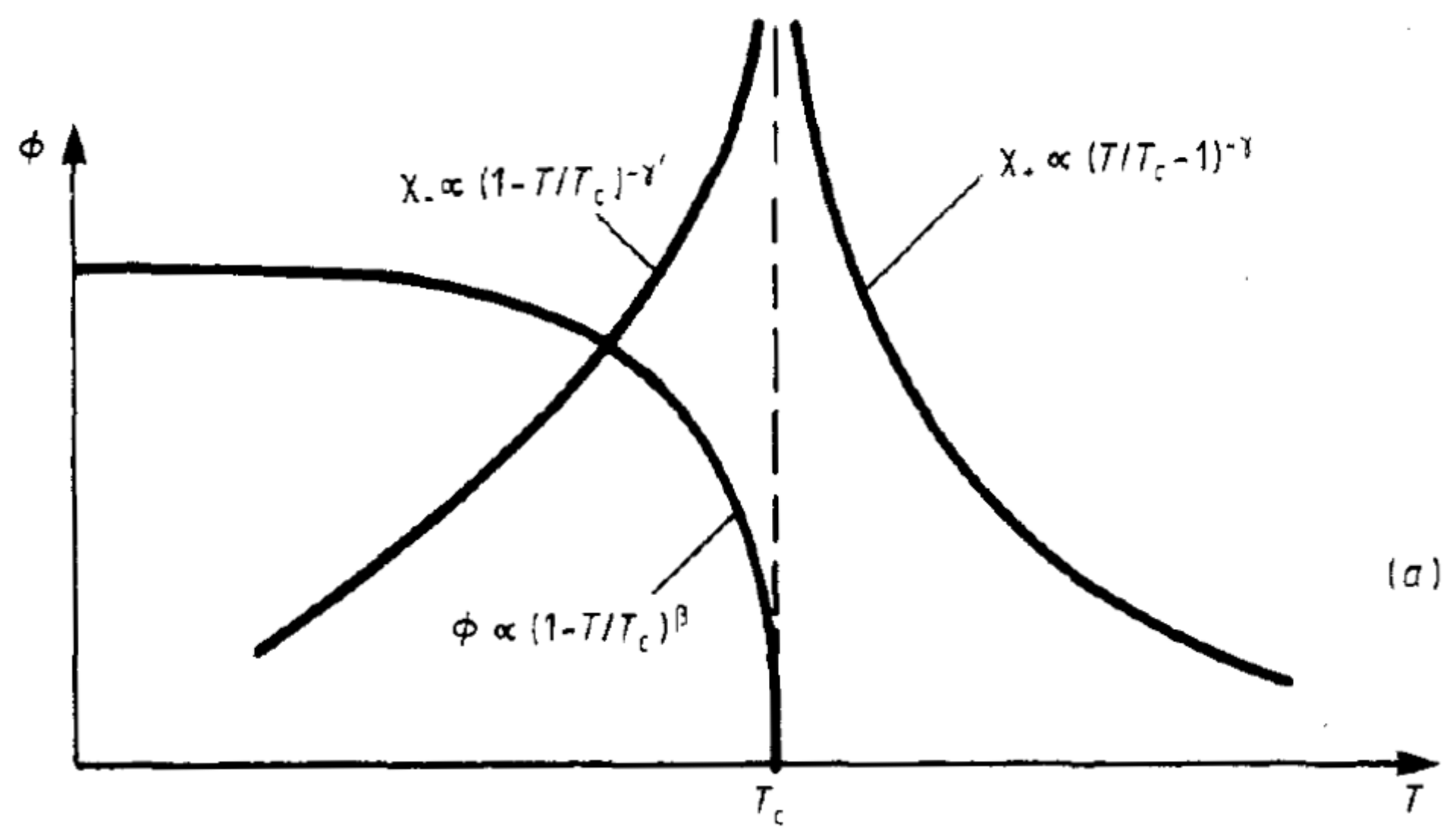




\section{An example: Random Boolean Networks (RBNs)}

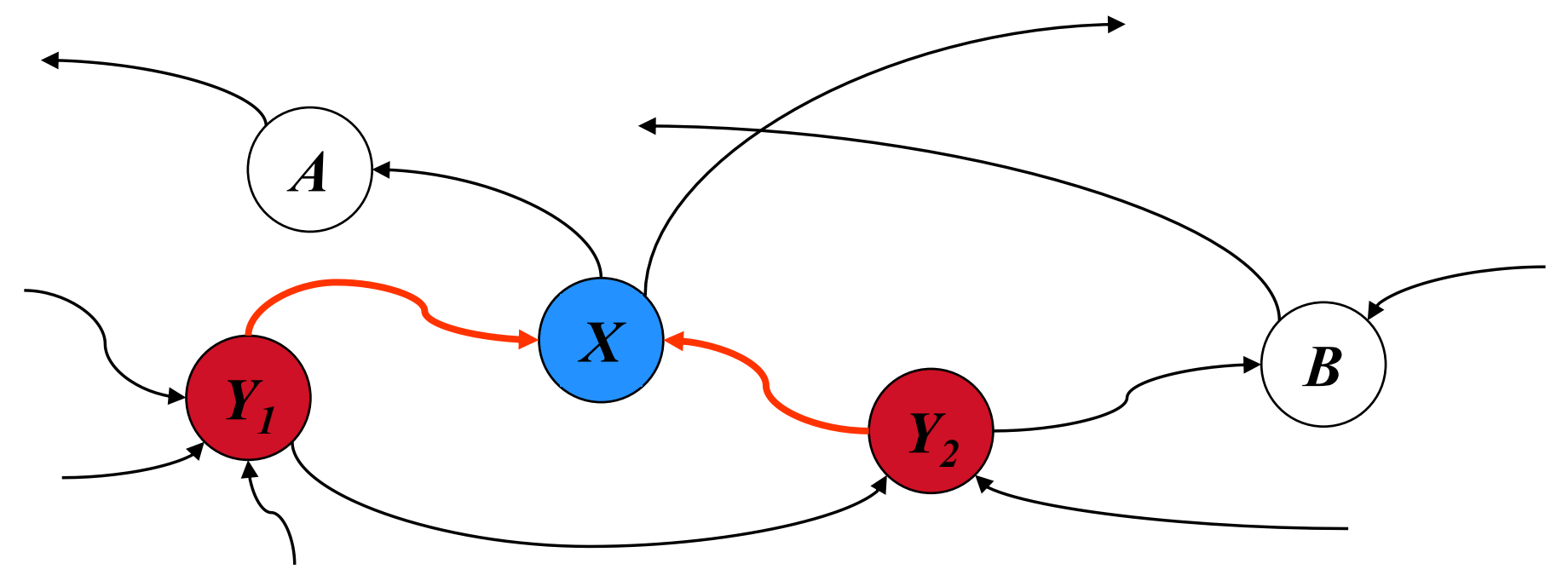

RBNs have:

- $\quad \mathrm{N}$ nodes in a directed structure

- which is determined at random from an average in-degree

$$
\bar{K}
$$

Each node has:

- Boolean states updated synchronously in discrete time

- $\quad$ update table determined at random, with some bias $r$ 


\section{Random Boolean Networks - phases of dynamics}

> Ordered

- Low connectivity (small $K$ ) or activity ( $r$ close to 0 or 1 )

- High regularity of states and strong convergence of similar global states in state space

> Chaotic

- High connectivity and activity

- Low regularity of states and divergence of similar global states

> Critical

- The "edge of chaos", separating ordered and chaotic phases

- Change at a node in the network spreads marginally

- Compromise between "stability" and "evolvability"

- Given bias $r$, can calculate $K$

$$
K_{c}=\frac{1}{2 r(1-r)}
$$




\section{Fisher Information: sensitivity}

> A way of measuring the amount of information that an observable random variable $X$ has about an unknown parameter $\theta$

$$
F(\theta)=\int_{x}\left(\frac{\partial \ln (p(x \mid \theta))}{\partial \theta}\right)^{2} p(x \mid \theta) d x
$$

- Fisher information is not a function of a particular observation, since the random variable $X$ is averaged out 


\section{Fisher Information and order parameters}

\section{$G\left(T, \theta_{i}\right)=U\left(S, \phi_{i}\right)-T S-\phi_{i} \theta_{i}$}

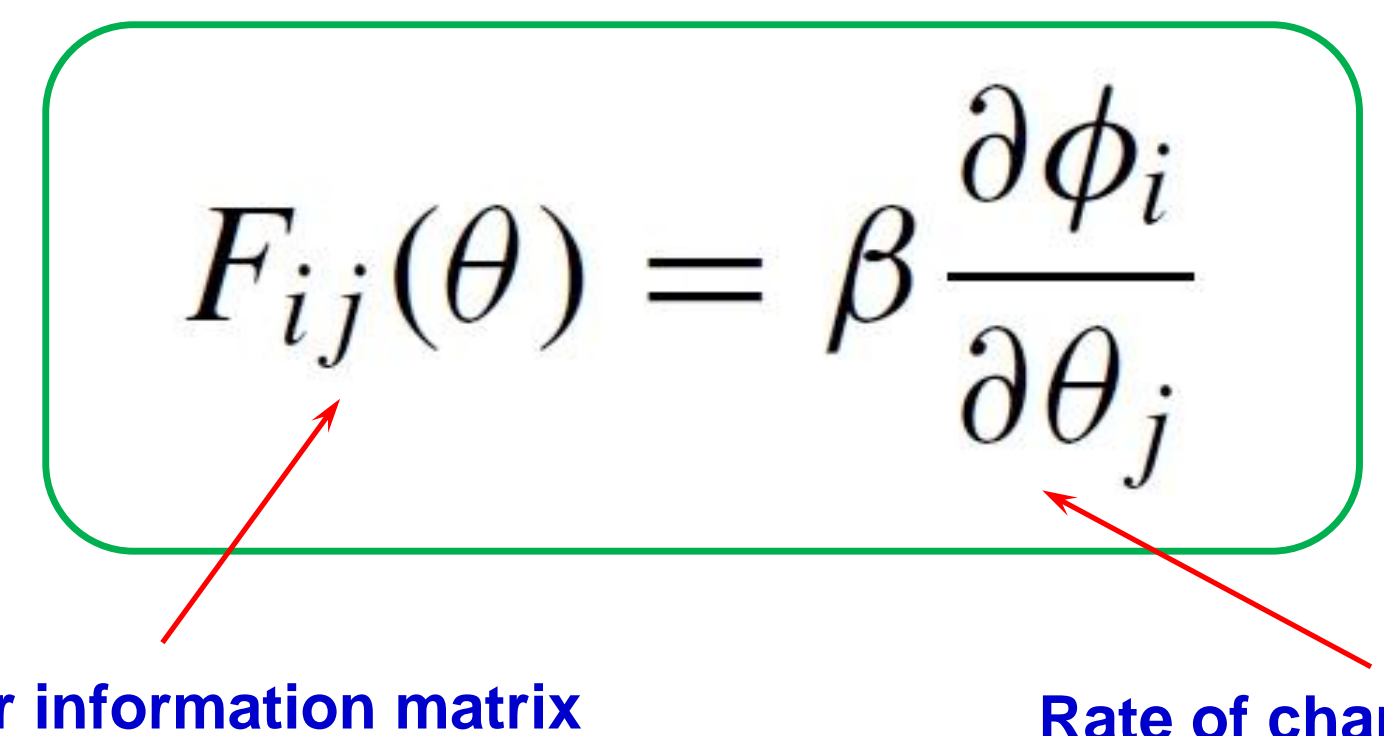

Fisher information matrix

Rate of change of the order parameter

M. Prokopenko, J. T. Lizier, O. Obst, and X. R. Wang, Relating Fisher information to order parameters, Physical Review E, 84, 041116, 2011. 


\section{Fisher Information - finite-size RBNs}

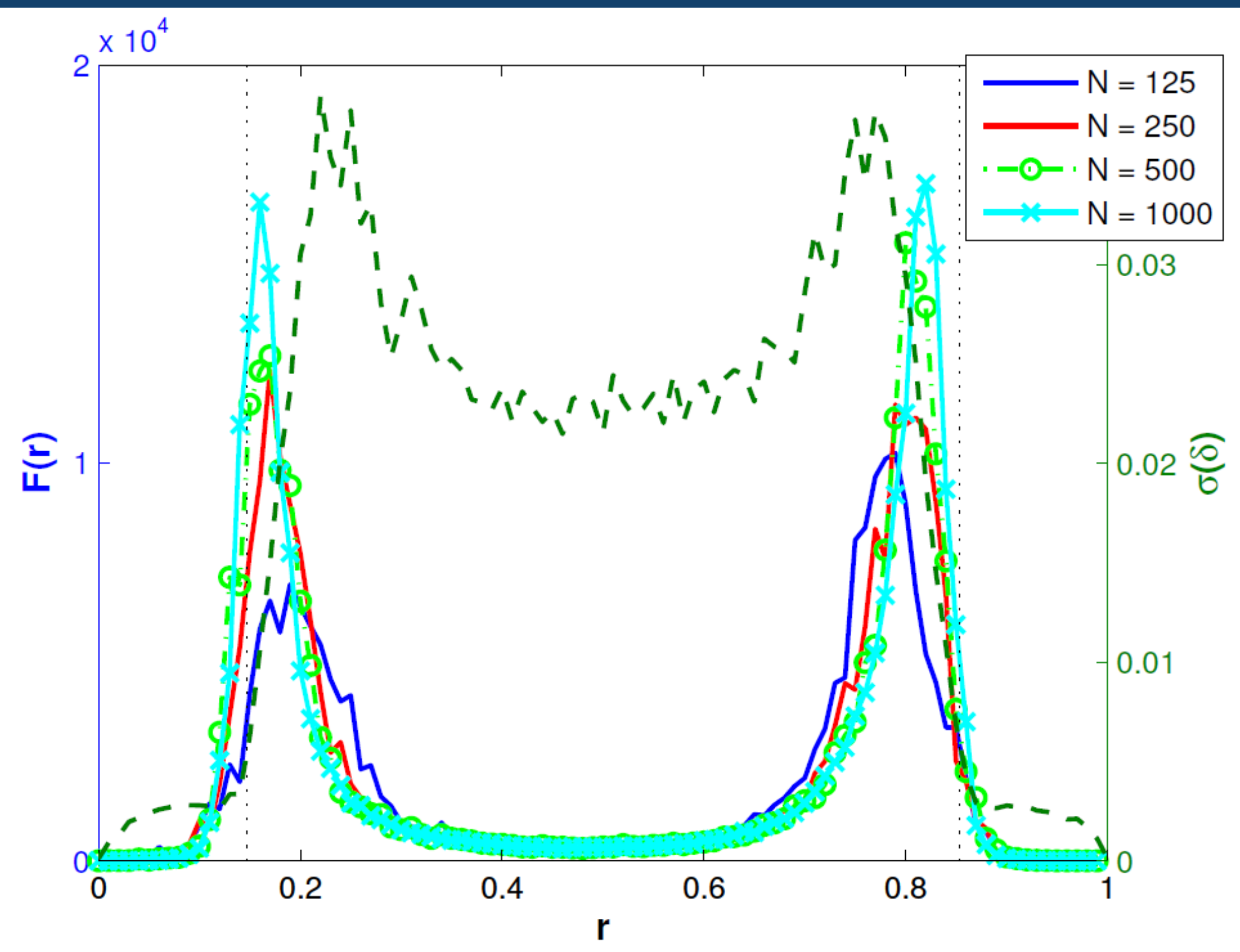




\section{Phase diagram - via Fisher information}

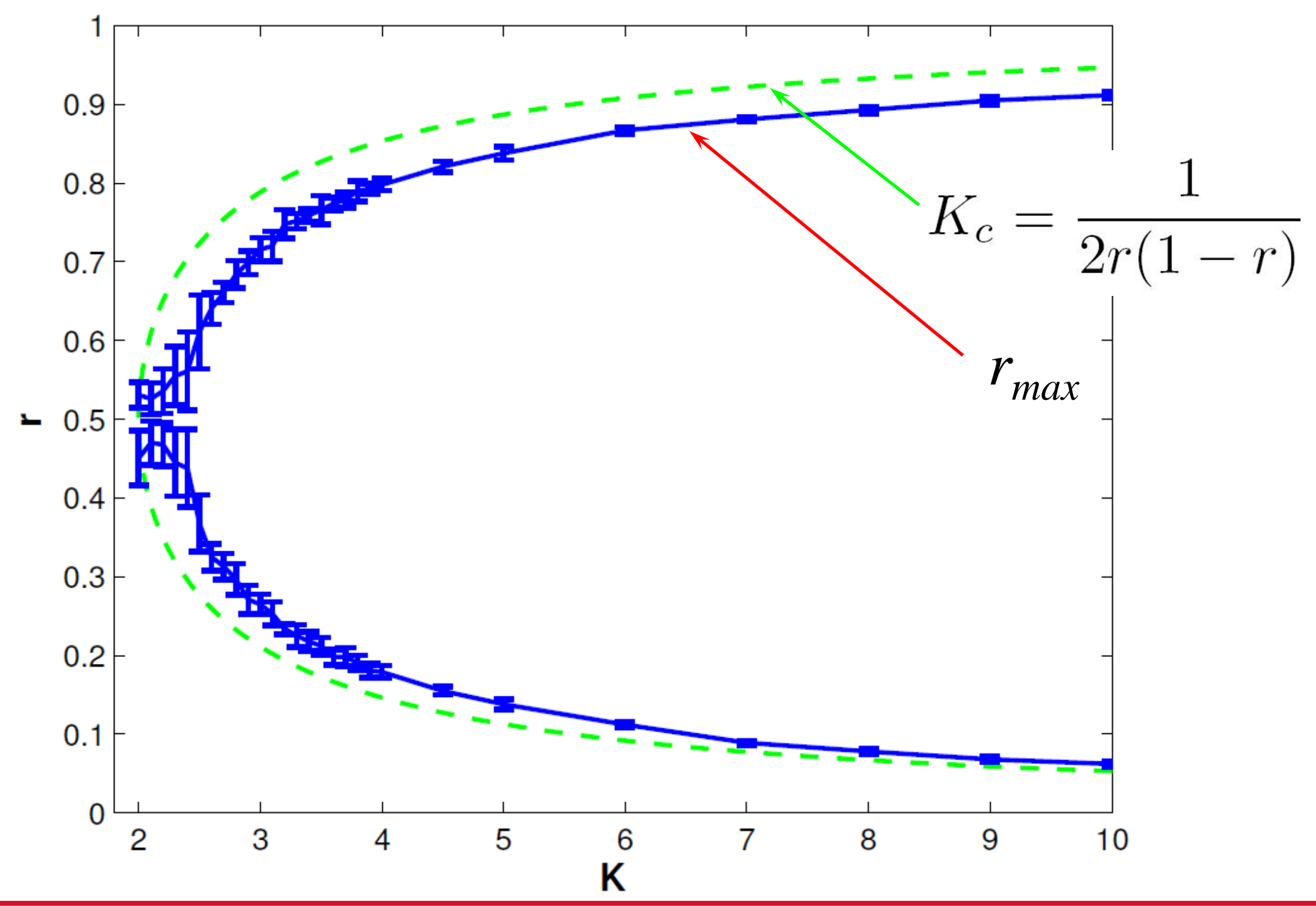




\section{A thermodynamic connection between} Fisher information and exported entropy

$$
G\left(T, \theta_{i}\right)=U\left(S, \phi_{i}\right)-T S-\phi_{i} \theta_{i}
$$

$F_{i j}(\theta)=\beta \frac{\partial \phi_{i}}{\partial \theta_{j}}$

$$
\Delta \sigma_{\exp }=-d\left(\frac{U\left(S, \phi_{i}\right)-\phi_{i} \theta_{i}}{T}\right)
$$

$$
\frac{\partial \Delta \sigma_{\text {exp }}}{\partial \theta_{i}}=\left(\frac{\partial^{2} S}{\partial \theta_{i}^{2}}-F\left(\theta_{i}\right)\right) d \theta_{i}
$$

Prokopenko, M., Einav, I. Information thermodynamics of near-equilibrium computation, Physical Review E, 91(6), 1-8, 2015. 


\section{Difference between two curvatures}

$$
\frac{\partial \Delta \sigma_{e x p}}{\partial \theta_{i}}=\left(\frac{\partial^{2} S}{\partial \theta_{i}^{2}}-F\left(\theta_{i}\right)\right) d \theta_{i}
$$




\section{Generic difference between two curvatures}

$$
\frac{d^{2} \mathbb{S}}{d^{2} \theta}=\frac{d^{2} S}{d^{2} \theta}-F(\theta)
$$

$$
\frac{\partial \Delta \sigma_{e x p}}{\partial \theta_{i}}=\left(\frac{\partial^{2} S}{\partial \theta_{i}^{2}}-F\left(\theta_{i}\right)\right) d \theta_{i}
$$




\section{Generic difference between two curvatures}

$$
\frac{d^{2} \mathbb{S}}{d^{2} \theta}=\frac{d^{2} S}{d^{2} \theta}-F(\theta)
$$
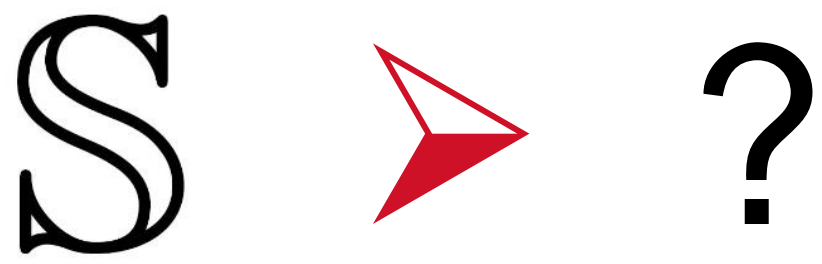


\section{Generic difference between two curvatures}

$$
\frac{d^{2} \mathbb{S}}{d^{2} \theta}=\frac{d^{2} S}{d^{2} \theta}-F(\theta)
$$

$$
\frac{d^{2}\left\langle\beta U_{\text {gen }}\right\rangle}{d \theta^{2}}=\frac{d^{2} S}{d \theta^{2}}-F(\theta)
$$

E. Crosato, R. Spinney, R. Nigmatullin, J. T. Lizier, M. Prokopenko, Thermodynamics of collective motion near criticality, 2017. 


\section{Revisiting our motivating questions...}

Chris Langton, "Computation at the edge of chaos: Phase transitions and emergent computation" (1991):

- how can emergence of computation be explained in a dynamic setting?

- how is it related to complexity of the system in point?

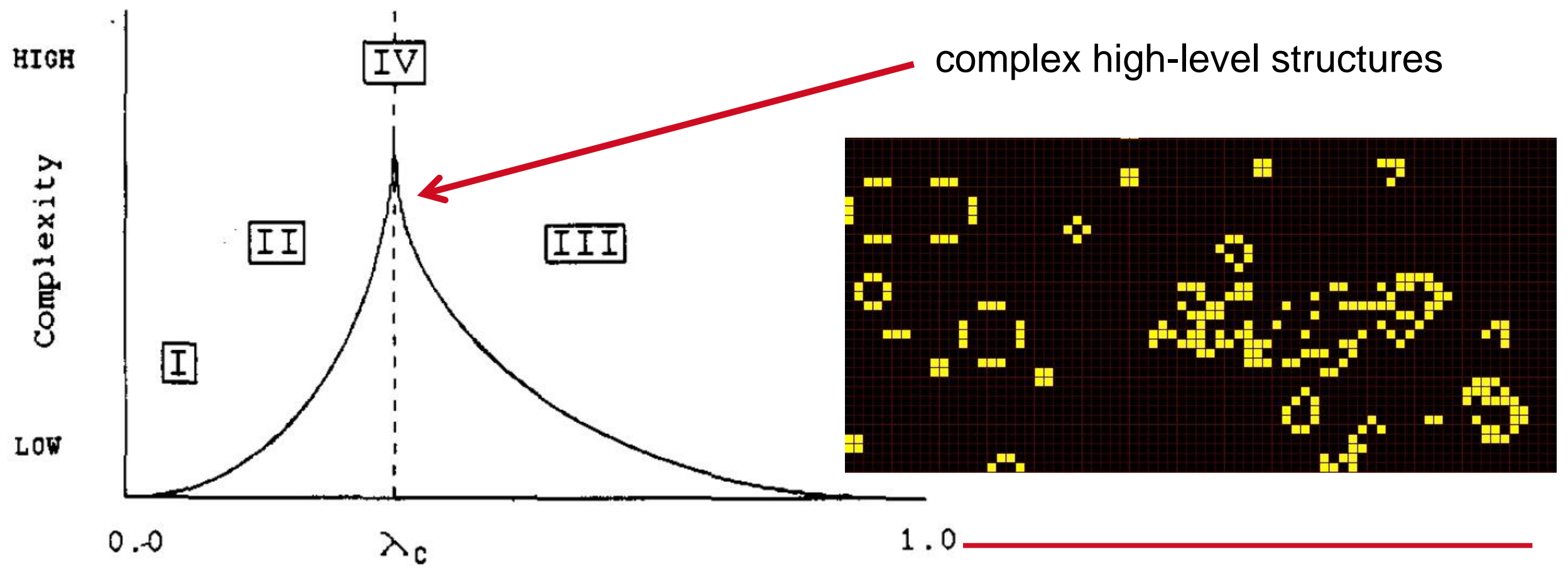




\section{Computation: sensitivity vs uncertainty}

$$
\frac{d^{2}\left\langle\beta U_{\text {gen }}\right\rangle}{d \theta^{2}}=\frac{d^{2} S}{d \theta^{2}}-F(\theta)
$$
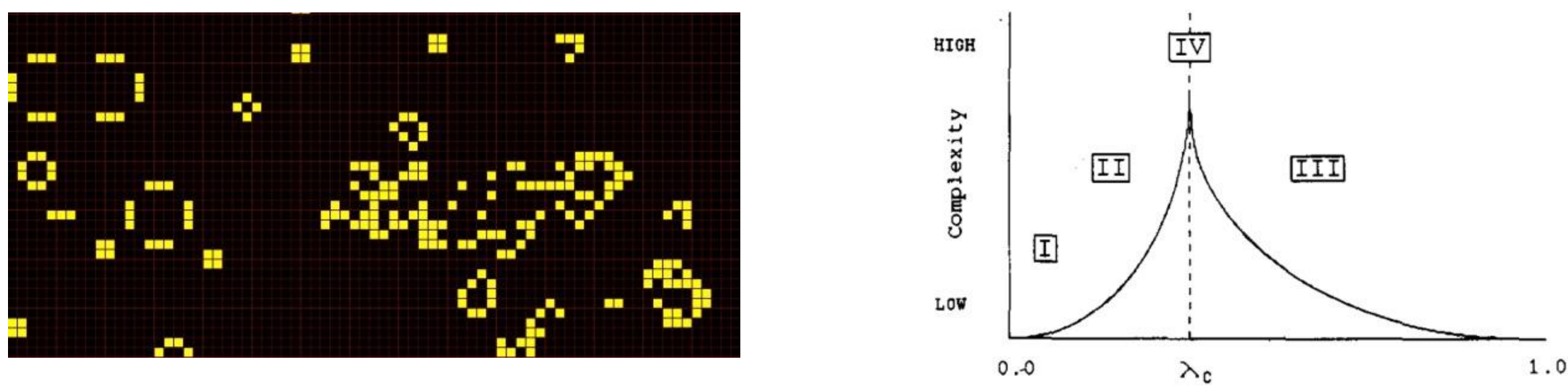


\section{Collective motion}

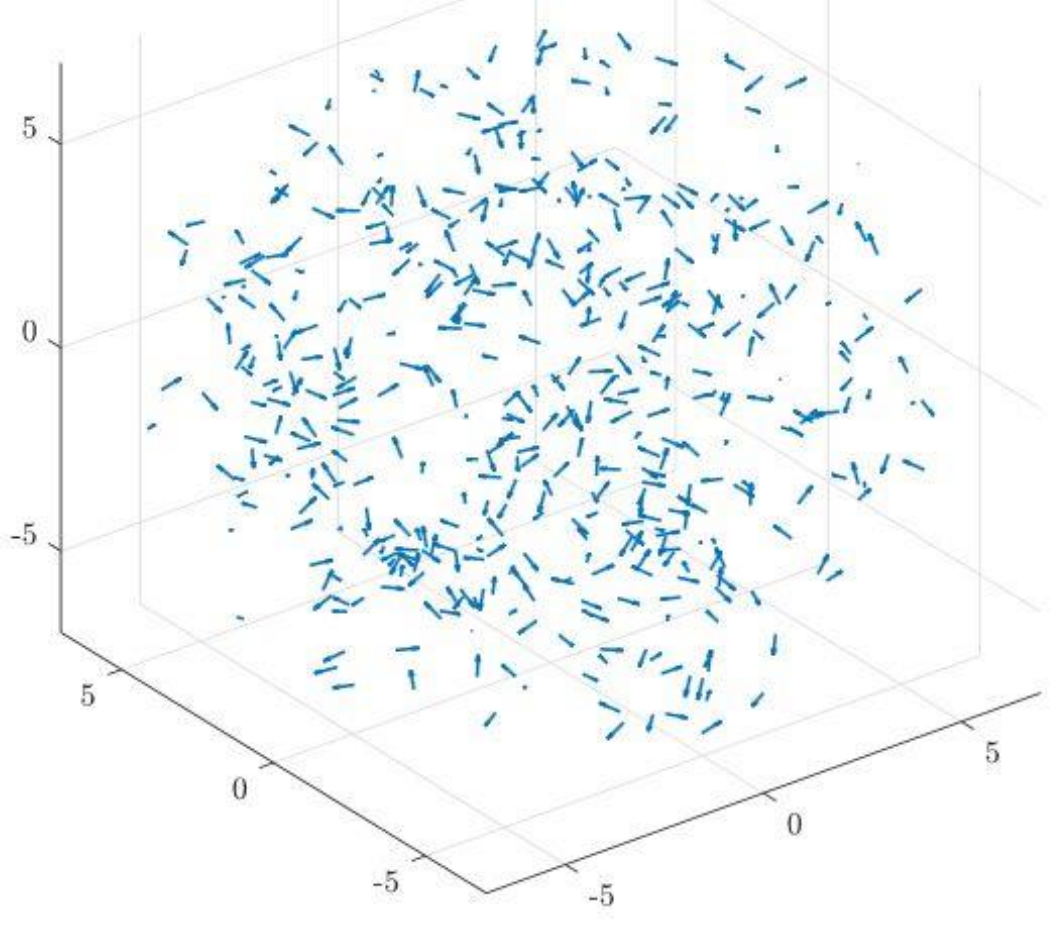

(a) $J=0.001, n_{c}=20$

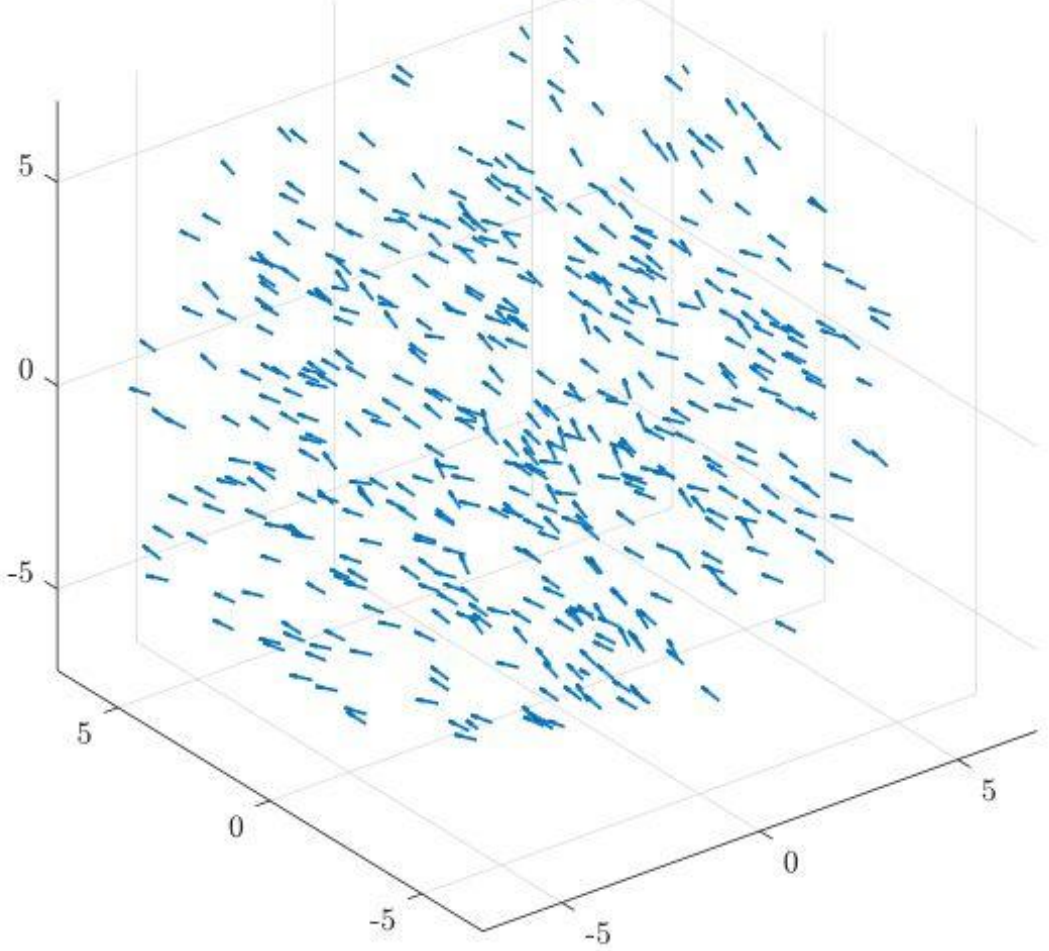

(b) $J=0.2, n_{c}=20$

E. Crosato, R. Spinney, R. Nigmatullin, J. T. Lizier, M. Prokopenko, Thermodynamics of collective motion near criticality, 2017. 


\section{Fisher information in collective motion}

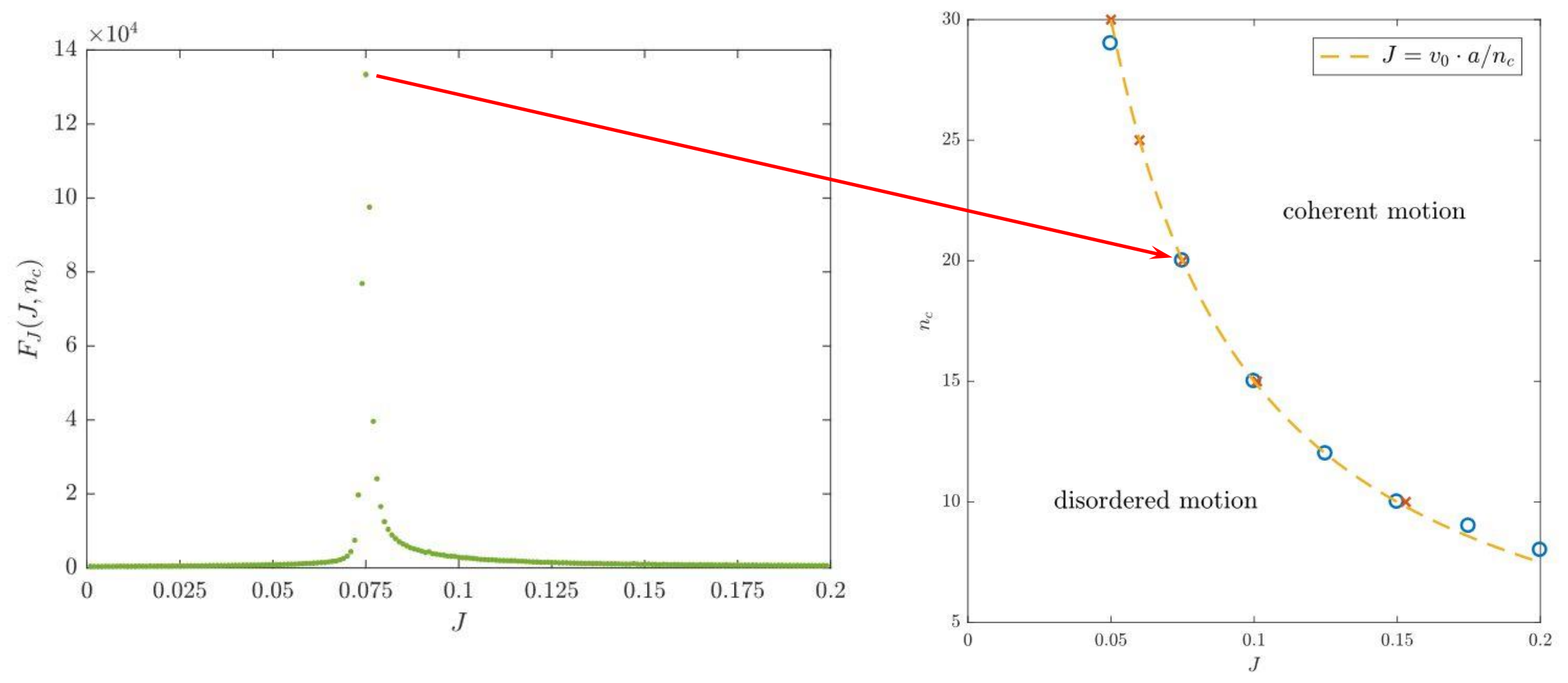

E. Crosato, R. Spinney, R. Nigmatullin, J. T. Lizier, M. Prokopenko, Thermodynamics of collective motion near criticality, 2017 . 


\section{Balance between sensitivity and uncertainty}

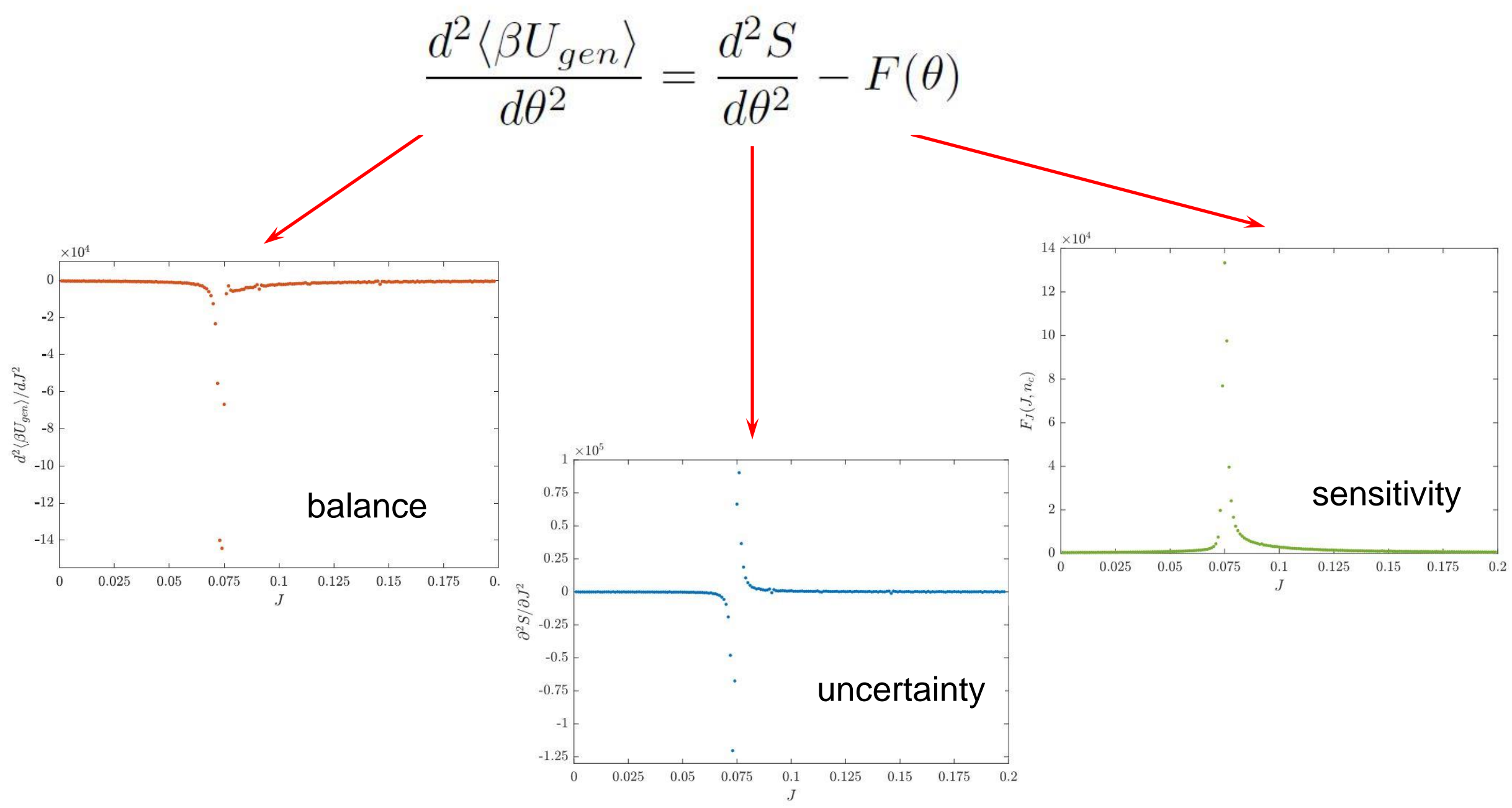

E. Crosato, R. Spinney, R. Nigmatullin, J. T. Lizier, M. Prokopenko, Thermodynamics of collective motion near criticality, 2017 . 


\section{Conclusions}

$>$ Edge of chaos: balance between order and chaos

$>$ Sensitivity of computation: Fisher information

$>$ Uncertainty of computation: entropy curvature

$>$ Balance: uncertainty vs sensitivity 
Master of Complex Systems Anticipate, control and manage the complexity of the unexpected

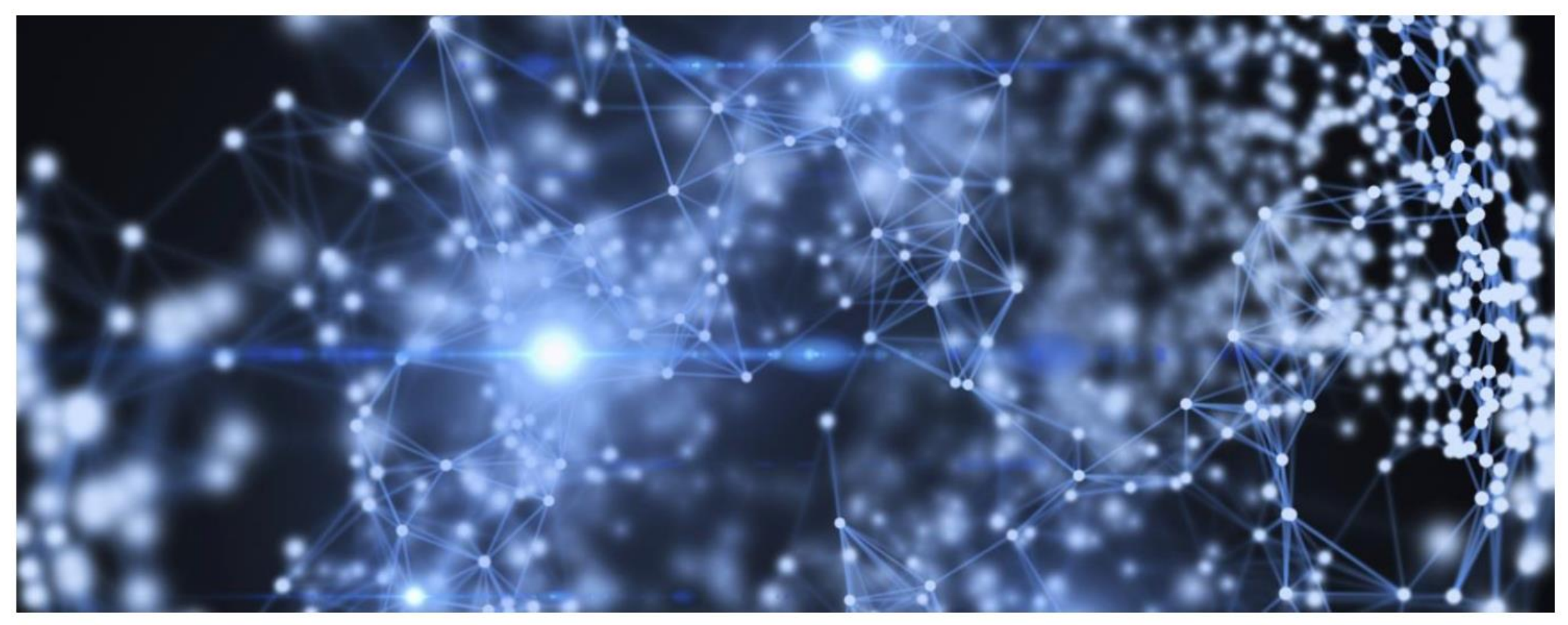

http://sydney.edu.au/courses/master-of-complex-systems 